\title{
Chordin-like protein 1 promotes neuronal differentiation by inhibiting bone morphogenetic protein-4 in neural stem cells
}

\author{
WEI-LU GAO ${ }^{1}$, SHENG-QUAN ZHANG ${ }^{2}$, HUI ZHANG ${ }^{1}$, BO WAN ${ }^{3}$ and ZONG-SHENG YIN ${ }^{1}$ \\ ${ }^{1}$ Department of Orthopaedics, The Geriatric Institution, First Affiliated Hospital of Anhui Medical University, Hefei, \\ Anhui 230022; ${ }^{2}$ Department of Biochemistry and Molecular Biology, Anhui Medical University, Hefei, Anhui 230032; \\ ${ }^{3}$ Department of Orthopaedics, The Third People's Hospital of Hefei, Anhui 230022, P.R. China
}

Received October 25, 2012; Accepted February 1, 2013

DOI: $10.3892 / \mathrm{mmr} .2013 .1310$

\begin{abstract}
In the present study, the effects of chordin-like protein 1 (CHRDL1) overexpression, together with bone morphogenetic protein-4 (BMP-4) treatment, on the differentiation of rat spinal cord-derived neural stem cells (NSCs) was investigated. Adult rat spinal cord-derived NSCs were cultured in serum-free medium. The recombined eukaryotic expression vector pSecTag2/Hygro B-CHRDL1 was transfected into adult rat spinal cord-derived NSCs using a lipid-based transfection reagent and protein expression was assessed by western blot analysis. Differentiation of transfected NSCs following BMP-4 treatment was determined by immunocytochemistry. The percentage of microtubule-associated protein-2 (MAP-2)-positive cells in the BMP-4-treated (B) group was found to be significantly lower compared with that in the non-transfected control (N) group. The percentage of MAP-2-positive cells in the pSecTag2/Hygro B-CHRDL1-transfected, BMP-4-treated group was identified to be significantly higher compared with that in group $\mathrm{B}$, however, no significant difference was observed between group $\mathrm{N}$ and the transfected, non-BMP-4-treated control group. The current study indicates that CHRDL1 protein antagonizes BMP-4 activity and induces spinal cord-derived NSCs to differentiate into neurons.
\end{abstract}

\section{Introduction}

In individuals who sustain a serious spinal cord injury, $<1 \%$ experience complete recovery of neurological function and a number of these injuries result in partial or complete paralysis (1). These injuries reduce the quality of life of the

Correspondence to: Dr Zong-Sheng Yin, Department of Orthopaedics, The Geriatric Institution, First Affiliated Hospital of Anhui Medical University, 218 Jixi Road, Anhui, Hefei 230022, P.R. China

E-mail: yinzs1961@163.com

Key words: chordin-like protein 1, neural stem cells, cell differentiation, gene expression individuals who may become a burden to society. Therefore, therapeutic interventions for spinal cord injury (SCI) have become one of the most intensely studied areas in neuroscience over the last few decades. Several cell transplantation studies have demonstrated some success in the treatment of SCI, however, exogenous neural stem cells (NSCs), which retain marked neurogenic activities in vitro, are associated with low levels of differentiation when grafted into the spinal cord $(2,3)$.

An additional issue associated with the use of NSCs for the treatment of SCI is that differentiated, but unpurified, stem cell transplants from the embryo may cause tumor formation within the central nervous system (CNS) (4). In addition, the transplantation of xenograft cells requires the co-administration of corticosteroids during the experimental period to prevent cell rejection, which may complicate interpretation of the results $(5,6)$. The transplantation of exogenous NSCs also has ethical considerations, since these cells are derived from embryonic tissue (7). Together, these unresolved issues have slowed the development of exogenous NSC transplantation therapy (8).

It is now generally accepted that NSCs reside in specific regions of the adult mammalian CNS, including the ependymal region of the spinal cord as well as the subventricular zone, hippocampus and dentate gyrus of the brain $(9,10)$. In addition, the adult spinal cord is an abundant source of endogenous NSCs and these cells may undergo considerable levels of proliferation under physiological conditions, including SCI (11). Due to the restrictive environment of the contusive spinal cord, proliferative endogenous NSCs mainly differentiate into astrocytes and form astrocytic scars, which have no therapeutic effect on the re-establishment of neural pathways (12). However, it has been hypothesized that if this environmental restriction is relieved by specific manipulations, then endogenous NSCs may be able to supply new neurons, which in turn may contribute to the reconstruction of local neuronal circuitry and facilitate the regeneration of long-distance axonal tracts (13-15). To date, strategies that manipulate endogenous NSCs in the context of SCI have not been explored.

Bone morphogenetic proteins (BMPs) play critical roles in the determination of NSC fate. Previous studies have demonstrated that BMP-4 promotes the differentiation of NSCs into astrocytes $(16,17)$. BMP-4 antagonists prevent the activation 
of BMP-4 receptors and thus alter the fate of NSCs toward neurogenisis $(18,19)$. Chordin-like protein 1 (CHRDL1), also known as neurogenesin-1 ( $\mathrm{Ng} 1)$, ventroptin and neuralin-1, is a BMP receptor antagonist that was originally identified in Xenopus $(20,21)$. CHRDL1 is predominantly secreted by astrocytes and hypothesized to be involved in adult neurogenesis (21). CHRDL1 has previously been shown to induce neuronal differentiation in brain-derived NSCs in culture (20).

The aforementioned studies indicate that CHRDL1 may play a key role in neurogenesis in the adult hippocampus. However, it is not yet known whether CHRDL1 also alters the fate of spinal cord-derived NSCs from gliogenesis to neurogenesis. Thus, in the current study, we assessed whether BMP-4 inhibition by CHRDL1 promotes the differentiation of spinal cord-derived NSCs into neurons in vitro.

\section{Materials and methods}

Animals. Three 7-day-old Sprague-Dawley (SD) rats and one adult SD rat (225 g) were used in this study. Animal care and use was conducted at the Experimental Animal Center of Anhui Medical University under a protocol approved by the Institutional Animal Care and Use Committee.

Construction of recombinant expression vector. One 7-day-old rat was euthanized by cervical dislocation and the cerebrum was rapidly excised in a sterile container on ice. The hippocampus was then separated. Total RNA was extracted with TRIzol (Invitrogen Life Technologies, Carlsbad, CA, USA). The primers used were designed with HindIII and XhoI sites and prepared in accordance with the published CHRDL1 gene sequence (Gene ID: 363455) as follows: 5' primer: 5'-CCC AAG CTT CCA GTA AAA CAC TCA GAG ACA TAT-3'; 3' primer: 5'-CCG CTC GAG AAC AGT GGT CCT TTT CAG GTC TCT C-3'. Primers were provided by Takara Bio, Inc. (Shiga, Japan). Reverse transcription (RT) was performed using the RNA polymerase chain reaction (PCR) kit (AMV, v3.0; Takara Bio, Inc.) according to the manufacturer's instructions. PCR experiments were performed under the following conditions: denaturation at $94^{\circ} \mathrm{C}$ for $5 \mathrm{~min}$; amplification for 35 cycles of $94^{\circ} \mathrm{C}$ for $40 \mathrm{sec}$, $55^{\circ} \mathrm{C}$ for $40 \mathrm{sec}$ and $70^{\circ} \mathrm{C}$ for $90 \mathrm{sec}$; and extension at $72^{\circ} \mathrm{C}$ for 5 min. Glyceraldehyde 3-phosphate dehydrogenase (GAPDH) was used as an internal control.

The DNA fragment of the expected molecular size was purified using the AxyPrep DNA Gel Extraction kit (AxyGen, Inc., Union City, CA, USA) and cloned into the pSecTag2/Hygro B vector (Invitrogen Life Technologies) for DNA sequencing. DNA sequencing was performed by Sangon Biotech (Shanghai, China) and the data were compared with the GenBank database using the BLAST program. The confirmed rat CHRDLI gene and pSecTag2/Hygro B vector were digested with HindIII and XhoI restriction enzymes (Takara Bio, Inc.), respectively. The two digested products were then incubated with T4 DNA ligase (Takara Bio, Inc.) at $16^{\circ} \mathrm{C}$ for $6 \mathrm{~h}$ to ligate the DNA. The constructed plasmid was transformed into $E$. coli JM109 cells for amplification and the plasmid was designated pSecTag2/Hygro B-CHRDL1. The plasmid was then isolated using the AxyPrep Plasmid Miniprep kit (AxyGen, Inc.,) and confirmed by PCR screening and restriction enzyme digestion analysis. Three rats were used in three experimental replicates.
Isolation, culture and identification of NSCs from adult rat spinal cord. One adult rat was euthanized by intraperitoneal administration of sodium pentobarbital. Under aseptic conditions, the spinal cord was removed and immediately immersed in chilled normal saline solution. Next, the primary cultures were prepared as described previously (22). The isolated cell pellet was resuspended in Dulbecco's modified Eagle's medium (DMEM)/F12 (1:1) supplemented with 2\% B27 (all Invitrogen Life Technologies), $20 \mathrm{ng} / \mathrm{ml}$ epidermal growth factor (EGF), $20 \mathrm{ng} / \mathrm{ml}$ basic fibroblast growth factor (bFGF; both PeproTech, Rocky Hill, NJ, USA), 4 mmol/l glutamine and $1 \%$ penicillin/streptomycin. Cell suspensions were cultured in an incubator at $37^{\circ} \mathrm{C}$ and $5 \% \mathrm{CO}_{2}$. The media were replaced with a half-dose of fresh medium every 2-3 days. Following cell culture for $\sim 6$ days, the proliferation of cell clusters was observed. Cells were passaged every 6-7 days by mechanical separation.

Following the second passage, cells were cultured for 5 days and the observed neurospheres were plated on poly-L-lysine coated glass coverslips placed in 6-well plates and cultured for an additional $24 \mathrm{~h}$. Immunocytochemical (ICC) analysis was then performed to specifically detect NSCs. To induce differentiation, the neurospheres from passage 3 were dissociated into single cells, plated on poly-L-lysine coated glass coverslips and grown in a medium composed of DMEM/F12, 2\% B27, 10\% fetal bovine serum (FBS; Invitrogen Life Technologies) and antibiotics, without EGF or bFGF. Media were changed every 2 days for $\sim 6$ days. On day 7, ICC analysis was performed to detect astrocytes and neurons.

Transfection of adult spinal cord-derived NSCs. For the transfection experiments, cells were divided into the following four groups: pSecTag2/Hygro B-CHRDL1 transfection alone (C), addition of BMP-4 alone (B), pSecTag2/Hygro B-CHRDL1 transfection and addition of BMP-4(G) and Lipofectamine 2000 (Invitrogen Life Technologies) alone as a non-transfected control $(\mathrm{N})$.

The pSecTag2/Hygro B-CHRDL1 plasmid was transfected into NSCs using Lipofectamine 2000 according to the manufacturer's instructions. Prior to transfection, the neurospheres were isolated to form single-cell suspensions by mechanical separation; $4-8 \times 10^{5}$ cells were resuspended in $500 \mu \mathrm{l}$ growth medium without antibiotics and seeded into each well of a 6-well plate. For groups C and G, $4.0 \mu \mathrm{g}$ pSecTag2/Hygro B-CHRDL1 plasmid DNA was used. Following a 24-h incubation period, NSCs were passaged, 1:5, into fresh growth medium containing $20 \mathrm{ng} / \mathrm{ml} \mathrm{EGF,} 20 \mathrm{ng} / \mathrm{ml}$ bFGF, $2 \%$ B27 and 1\% penicillin/streptomycin. The following day, fresh growth medium supplemented with $200 \mu \mathrm{g} / \mathrm{ml}$ hygromycin B was added. After incubation for 5 days, the supernatant was collected for western blot analysis.

NSCs were then passaged in a medium composed of $\mathrm{DMEM} / \mathrm{F} 12,2 \% \mathrm{~B} 27$ and $10 \% \mathrm{FBS}$, in the absence of EGF or bFGF. BMP-4 (10 ng/ml) was then added to the group B and G cultures. Finally, 5 days following the addition of BMP-4, a double ICC assay was performed to assess the differentiation of NSCs.

Western blot analysis. Western blot analysis was performed to assess the expression of CHRDL1, Smad5 and phosphorylated 
Smad5 proteins in the NSCs. Briefly, 5 days following the addition of hygromycin B, supernatant samples were collected and 5 days after addition of BMP-4, cell extract samples were collected. Mouse anti-c-myc, goat anti-Smad5 (both 1:500; Santa Cruz Biotechnology, Santa Cruz, CA, USA), rabbit anti-phospho-Smad1/5 (1:200) and mouse anti- $\beta$-actin (1:300; both Cell Signaling Technology, Beverly MA, USA) were used as primary antibodies. Goat anti-mouse IgG antibody conjugated to horseradish peroxidase (HRP), rabbit anti-goat and goat anti-rabbit HRP-conjugated antibodies were used to reveal protein labeling. Membranes were then evaluated using an electrochemiluminesence western blot kit (Pierce Biotechnology, Inc., Rockford, IL, USA) according to the manufacturer's instructions.

Immunocytochemistry. Cells were grown on coverslips and fixed for $30 \mathrm{~min}$ at room temperature with $4 \%$ paraformaldehyde in $0.01 \mathrm{~mol} / 1 \mathrm{PBS}$ and then washed three times with $0.01 \mathrm{~mol} / 1 \mathrm{PBS}$ ( $\mathrm{pH} 7.4$ ). The cells were then permeabilized with $0.3 \%$ Triton $\mathrm{X}-100$ for $30 \mathrm{~min}$ at room temperature and then washed three times with $0.01 \mathrm{~mol} / 1 \mathrm{PBS}$. Non-specific interactions were blocked by incubating the cells in $5 \%$ normal goat serum in $0.01 \mathrm{~mol} / 1 \mathrm{PBS}$ for $40 \mathrm{~min}$ at room temperature. The cells were then incubated overnight at $4{ }^{\circ} \mathrm{C}$ with the following primary antibodies: mouse anti-nestin, anti-microtubule-associated protein-2 (MAP-2; both 1:200; Santa Cruz Biotechnology) and rabbit anti-glial fibrillary acidic protein (GFAP; 1:200; Abcam, Cambridge, MA, USA). The following day, cells were washed and incubated with corresponding secondary antibodies for $1 \mathrm{~h}$ at room temperature: fluorescein isothiocyanate-conjugated goat anti-mouse $\mathrm{IgG}$, tetraethyl rhodamine isothiocyanate-conjugated goat anti-rabbit IgG (both 1:100; Santa Cruz Biotechnology). When appropriate, Hoechst 33342 (Sigma-Aldrich, St. Louis, MO, USA) was used at a concentration of $10 \mu \mathrm{g} / \mathrm{ml}$ for $10 \mathrm{~min}$ to label cell nuclei. Fluorescent images were captured using a fluorescent microscope system (Nikon, Tokyo, Japan).

Statistical analysis. The in vitro cell number in each of the four experimental groups was estimated by counting 5 fields/coverslip. Neuron purity was determined by the ratio of MAP-2-positive cells to Hoechst 33342-positive cells. Comparisons were performed using the $\chi^{2}$ test. All data were analyzed with SPSS 11.0 software (SPSS, Inc., Chicago, IL, USA). $\mathrm{P}<0.05$ was considered to indicate a statistically significant difference.

\section{Results}

Molecular cloning of CHRDL1 and construction of eukaryotic vectors. RT-PCR products were analyzed by agarose gel electrophoresis in the presence of ethidium bromide. A $1.3-\mathrm{kb}$ product band was detected of the expected molecular size (Fig. 1A) and isolated, purified and cloned into the pSecTag2/Hygro B vector. Sequence analysis revealed that the inserted cDNA clone was 1,275 bp and was identical to the reported rat CHRDL1 sequence determined using the BLAST program. The cDNA clone corresponding to the rat CHRDL1 sequence was then sub-cloned into pSecTag2/Hygro B and confirmed by PCR screening and double restriction enzyme analysis (Fig. 1B).
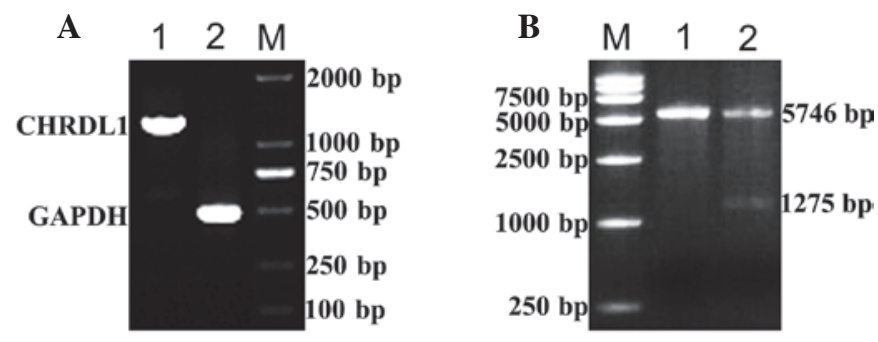

Figure 1. (A) Analysis of RT-PCR products. Lane 1, expected PCR product of $1.3 \mathrm{~kb}$; and 2, GAPDH. (B) Double restriction enzyme digestion products of pSecTag/Hygro B-CHRDL1. Lane 1, empty pSecTag/Hygro B; and 2, pSecTag/Hygro B cut with HindIII and XhoI, releasing the 1,275-bp CHRDL1 cDNA insert. M, DNA marker; CHRDL1; chordin-like protein 1; GAPDH, glyceraldehyde 3-phosphate dehydrogenase; RT-PCR, reverse transcription polymerase chain reaction.

Identification of adult spinal cord NSCs. At day 1 of culture, the majority of the cells were observed to be floating in the medium and free-floating with differing refractivity and the cells were three-dimensional by morphological observation. No neurospheres were observed in the medium. Following 3-4 days of culture, small free-floating spheres were observed. At day 7, the cells formed suspended, spherical clusters composed of numerous cells. The clusters were increased in size with weakly refractive centers (Fig. 2A). When the cells were resuspended by trituration and the suspension of single cells from the cell clusters was passaged, cell clusters formed again, indicating that these cell clusters may be neurospheres. Cells from the neurospheres were successfully passaged once per week through $\geq 16$ passages.

To determine the characteristics of the free-floating neurospheres, ICC analysis was performed. Specifically, neurospheres were examined for their expression of nestin, an NSC marker. As demonstrated in Fig. 2B and C, the spheres exhibited nestin positivity.

To examine the differentiation potential of adult spinal cord-derived NSCs, newly formed neurospheres from passage 3 were dissociated into single cells and transferred into medium containing $10 \%$ FBS. At day 6, cells were fixed and processed for ICC staining. The phenotypes of these new cells were determined using anti-GFAP and -MAP2 antibodies, which are specific markers for astrocytes and neurons, respectively. Immunofluorescent staining revealed that the majority of cells were GFAP positive (Fig. 2D) and a number were also MAP-2 positive (Fig. 2E). These results indicate that these cells were NSCs and had multidirectional differentiation potential.

Recombinant CHRDL1 protein reduces Smad5 protein levels. Western blot analysis revealed the presence of CHRDL1 protein overexpression in the transfected NSCs. As demonstrated in Fig. 3A, c-myc-tagged CHRDL1 protein was detected in the supernatant. A specific $46-\mathrm{kDa}$ protein band that corresponded to the protein was detected in groups $\mathrm{C}$ and $\mathrm{G}$, but not in groups $\mathrm{N}$ and $\mathrm{B}$. These results confirmed that the CHRDL1-myc fusion protein was successfully expressed in adult NSCs.

BMP-mediated induction of neuronal differentiation occurs via the Smad pathways, particularly the BMP-4/Smad5 signaling pathway (23). Analysis of cell extracts 5 days 


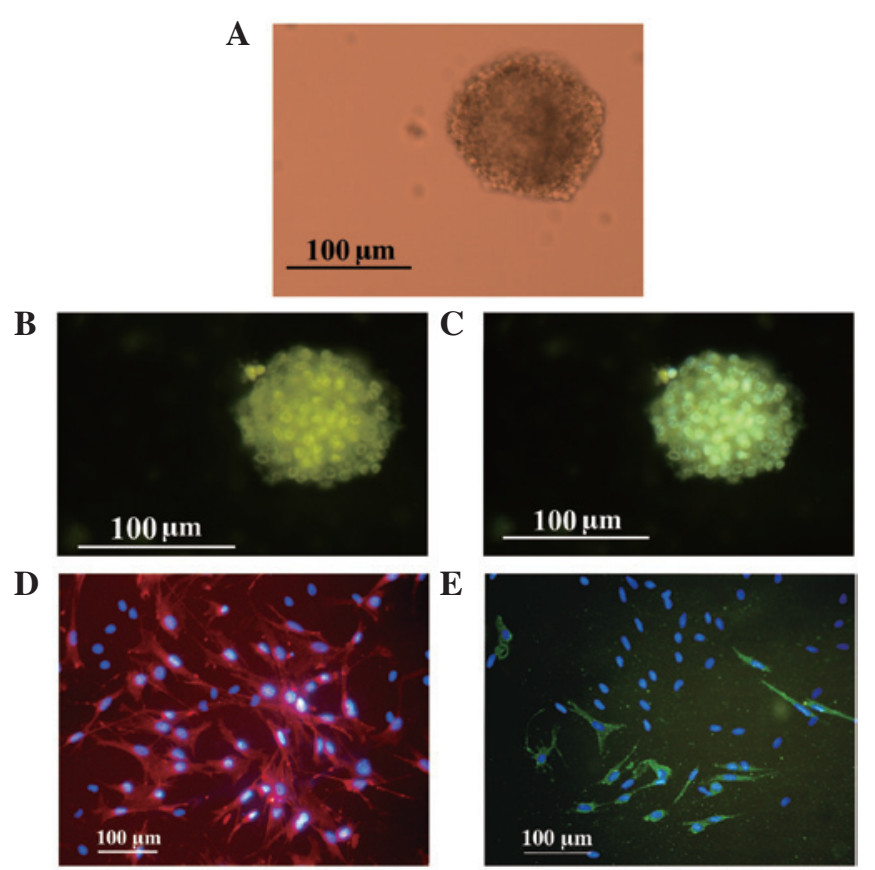

Figure 2. (A) Cultured NSCs. Cell cluster formation, following 6 day culture of the primary cells (inverted microscope). (B) Identification of adult NSCs in neurospheres using an anti-nestin specific monoclonal antibody, a cell-type specific marker of NSCs (FITC, green). (C) Merge of nestin (FITC, green) and Hoechst 33342 (blue). (D) Identification of cell differentiation. Astrocytes differentiated from neurospheres were GFAP positive and identified by immunofluorescent staining (TRITC, red) and Hoechst 33342 (blue). (E) Neurons differentiated from neuronspheres were MAP-2 positive (FITC, green) and Hoechst 33342 (blue). NSCs, neural stem cells; FITC, fluorescein isothiocyanate; GFAP, glial fibrillary acidic protein; TRITC, tetraethyl rhodamine isothiocyanate; MAP-2, microtubule-associated protein-2.

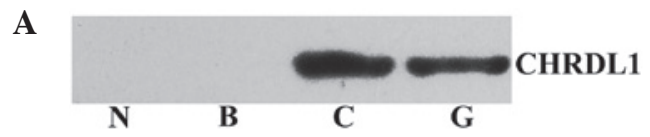

B

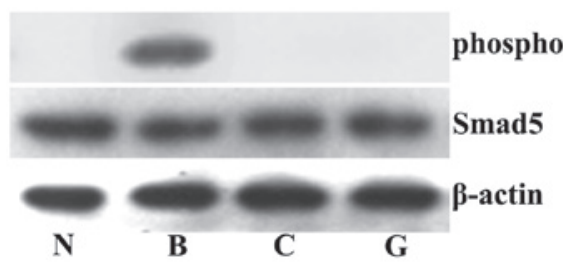

Figure 3. Western blot analysis of adult NSCs: N, control; C, transfected with CHRDL1; B, treated with BMP-4; and G, transfected with CHRDL1 and incubated with BMP-4. Expression of (A) recombinant CHRDL1 protein detected with an anti-Myc antibody and (B) phospho-Smad1/5. Phospho-Smad1/5 expression was markedly decreased in group $\mathrm{G} 5$ days following the addition of BMP-4, indicating that the BMP-4/Smad5 pathway was inhibited. $\beta$-actin and Smad5 were used as internal loading controls. Results are representative of at least three experiments. CHRDL1, chordin-like protein 1; NSCs, neural stem cells; BMP-4, bone morphogenetic protein-4.

following the addition of BMP-4 revealed a marked accumulation of phospho-Smad1/5 protein; the specific $60-\mathrm{kDa}$ protein band was detected in group B only (Fig. 3B). The absence of the specific protein band for groups $\mathrm{G}$ and $\mathrm{C}$ demonstrated that CHRDL1 inhibited the activation of Smad5 and that CHRDL1 does not affect the BMP-4/Smad5 pathway independently.
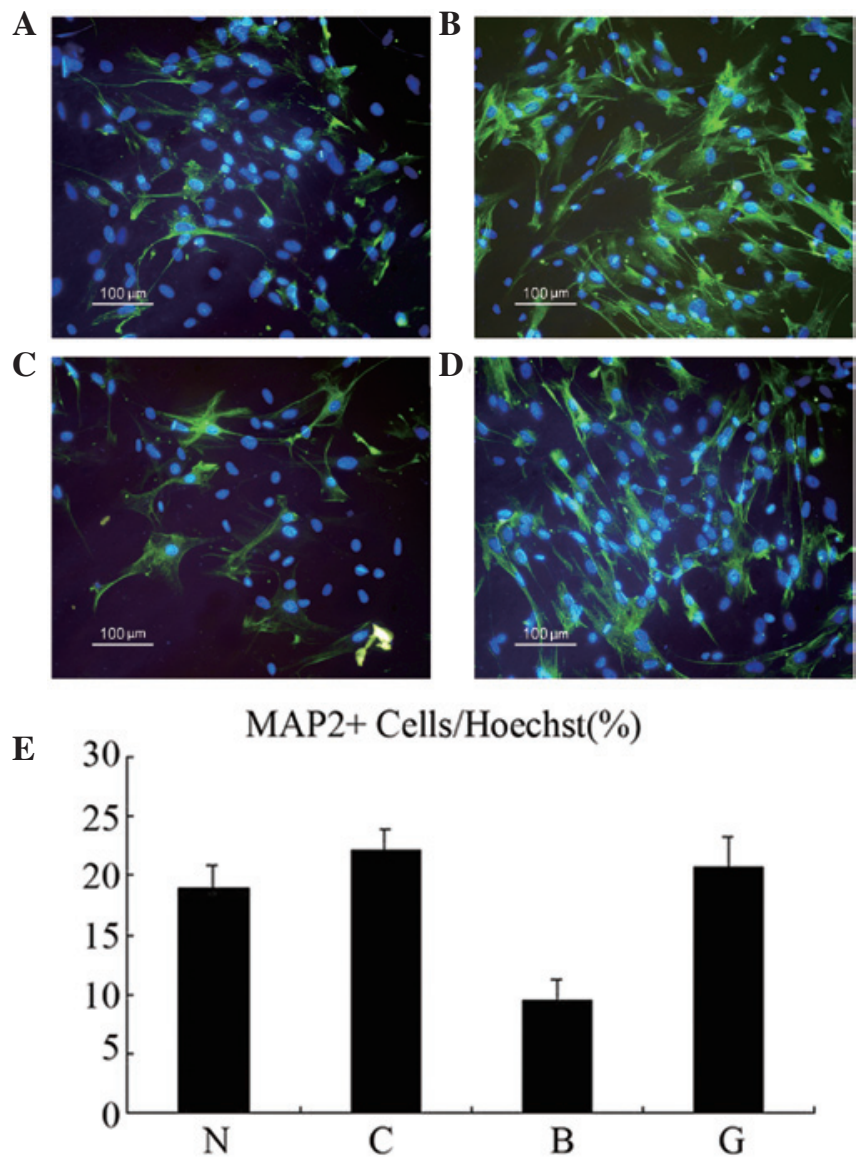

Figure 4. CHRDL1 promoted neuronal differentiation of adult NSCs: N, control; C, transfected with CHRDL1; B, treated with BMP-4; and G, transfected with CHRDL1 and incubated with BMP-4. MAP-2 (FITC, green) and Hoechst 33342 (blue) double-positive cells in groups (A) N, (B) C, (C) B and (D) G. (E) Percentage of MAP-2-positive cells among the total number of Hoechst 33342-positive cells. Groups: N, 18.92\%; C, 22.11\%; B, 9.59\%; and G, 20.65\%. CHRDL1, chordin-like protein 1; NSCs, neural stem cells; MAP-2, microtubule-associated protein-2; FITC, fluorescein isothiocyanate.

Recombinant CHRDL1 protein antagonizes BMP-4 and promotes neuronal differentiation of adult NSCs. The effect of CHRDL1 protein on adult NSCs was determined in the presence of BMP-4. Neurospheres were dissociated into single cell suspensions and transfected with CHRDL1 and/or treated with BMP-4, or treated with transfection reagent. At day 5 following the addition of BMP-4, a double immunofluorescence assay was performed (Fig. 4A-D). As revealed in Fig. 4E, the percentage of MAP-2-positive cells in group B (9.59\%) was found to be significantly lower than that in group $\mathrm{N}\left(18.92 \% ; \chi^{2}=5.400 ; \mathrm{P}<0.05\right)$. In addition, the percentage of MAP-2-positive cells in group G (20.65\%) was observed to be significantly higher than that in group B $\left(9.59 \% ; \chi^{2}=7.437 ; \mathrm{P}<0.05\right)$. No significant difference was identified between groups $\mathrm{N}(18.92 \%)$ and $\mathrm{C}\left(22.11 \% ; \chi^{2}=0.583\right.$; $\mathrm{P}>0.05)$. Similar results were obtained in three independent experiments. These results indicate that CHRDL1 expression antagonized BMP-4 and promoted the neuronal differentiation of adult NSCs in vitro.

\section{Discussion}

In the present study, CHRDL1 protein was ectopically overexpressed in rat spinal cord-derived NSCs and considerable 
levels of active CHRDL1 protein were secreted from the transfected NSCs into the culture medium. CHRDL1 transfection of adult rat spinal cord-derived NSCs was found to attenuate BMP-4 treatment-induced reductions in a number of the MAP-2-positive cells. This effect appears to be mediated by the BMP-4/Smad5 pathway.

BMPs participate in diverse processes, including neurogenesis $(24,25)$, the formation of anterior-posterior and dorsal-ventral axes and apoptosis (26). The BMP signaling pathway is highly conserved and vital to a variety of organ developmental pathways. However, the effect of BMPs on NSCs is extremely complicated as BMPs induce NSCs to differentiate into neurons or astrocytes. Studies have reported conflicting results concerning the effect of BMPs on NSCs, even when assessed in the same anatomical location or development phase (27-29). BMP-4 is expressed in the embryonic cortex (30), indicating a potential role in differentiation. Consistent with the hypothesis that BMP-4 may be significant in developmental differentiation, it has been observed to promote differentiation of spinal cord-derived NSCs towards an astrocyte fate (31). BMP inhibitors that bind directly to BMP and thereby prevent BMP activation, are potential factors in the determination of neural fate commitment. Neural inducers, including noggin, chordin and follistatin, do not have their own receptors on target cells, instead, these molecules act by binding to and inactivating BMP-4 (32).

Ueki et al (20) first cloned and characterized the secretory factor, CHRDL1, and revealed that it contained an N-terminal signal peptide, three cysteine-rich domains and shared similarities with chordin. The C-terminal segments were composed of amino acid sequences unique to CHRDL1 and the structural features of CHRDL1 indicated that CHRDL1 may bind BMP-4 at BMP-binding modules residing in cysteine-rich domains. The authors revealed that CHRDL1 antagonizes BMP-4 and alters the fate commitment of brain-derived NSCs from gliogenesis to neurogenesis. However, brain- and spinal cord-derived NSCs have distinct properties and growth requirements (33). Therefore, in the present study, the ability of CHRDL1 to promote the differentiation of spinal cord-derived NSCs into neurons was determined.

In the present study, the coding region of CHRDL1 cDNA was cloned without the putative signal peptide. The coding region of the cDNA was $1,275 \mathrm{bp}$ and contained three cysteine-rich domains that shared similarities with chordin. The gene was then sub-cloned into the pSecTag2/Hygro B vector, an expression vector designed for high-level expression and secretion in mammalian hosts. The vector contains the gene encoding $\beta$-lactamase, an $\mathrm{N}$-terminal murine $\mathrm{Ig} \kappa$ chain leader sequence for protein secretion and a C-terminal peptide containing the c-myc epitope followed by six tandem histidine residues that are used for detection and purification. These properties of the vector enabled us to transfect NSCs and generate secreted, tagged CHRDL1 protein in these cells. Restriction enzyme digestion and western blot analysis revealed that the CHRDL1 gene had been successfully cloned and expressed the protein in NSCs. The effects of CHRDL1 on the proliferation and differentiation of adult NSCs derived from the rat spinal cord in vitro were analyzed and a significant difference was identified between groups $\mathrm{B}$ and $\mathrm{N}$, indicating that BMP-4 inhibits NSC differentiation into neurons. In addition, a significant difference between groups $\mathrm{G}$ and $\mathrm{B}$ was observed, indicating that the CHRDL1 protein promotes the differentiation of NSCs in the presence of BMP-4. However, no statistical difference was identified between groups $\mathrm{G}$ and $\mathrm{B}$, which demonstrated that the CHRDL1 protein did not promote NSC differentiation into a neuronal fate independently. Neurosphere cultures from adult rat spinal cord revealed that BMP-4 inhibited NSC differentiation toward a neuronal fate. By contrast, inhibition of BMP-4 signaling by CHRDL1 markedly increased the ratio of neurons to total cell number and this effect was mediated by the BMP-4/Smad5 pathway.

In conclusion, the current study demonstrates that the CHRDL1 gene induces the differentiation of endogenous NSCs into neurons. These novel observations indicate that CHRDL1 may play a key role in adult spinal cord neurogenesis in mammals and provide neurogenic cues for endogenous NSCs.

\section{Acknowledgements}

The authors thank Guang-Wu Li who directed the separation of the hippocampus and Yu Chai, Dao-Jun Hu and Ren Zhao who provided experimental assistance. The present study was funded by the National Nature Science Foundation of China (no. 30772201).

\section{References}

1. Willerth SM and Sakiyama-Elbert SE: Cell therapy for spinal cord regeneration. Adv Drug Deliv Rev 60: 263-276, 2008.

2. Hill CE, Proschel C, Noble M, Mayer-Proschel M, Gensel JC, Beattie MS and Bresnahan JC: Acute transplantation of glial-restricted precursor cells into spinal cord contusion injuries: survival, differentiation and effects on lesion environment and axonal regeneration. Exp Neurol 190: 289-310, 2004.

3. Enzmann GU, Benton RL, Woock JP, Howard RM, Tsoulfas P and Whittemore SR: Consequences of noggin expression by neural stem, glial and neuronal precursor cells engrafted into the injured spinal cord. Exp Neurol 195: 293-304, 2005.

4. Roy NS, Cleren C, Singh SK, Yang L, Beal MF and Goldman SA: Functional engraftment of human ES cell-derived dopaminergic neurons enriched by coculture with telomerase-immortalized midbrain astrocytes. Nat Med 12: 1259-1268, 2006.

5. McDonald JW, Liu XZ, Qu Y, et al: Transplanted embryonic stem cells survive, differentiate and promote recovery in injured rat spinal cord. Nat Med 5: 1410-1412, 1999.

6. Ma H, Yu B, Kong L, Zhang Y and Shi Y: Transplantation of neural stem cells enhances expression of synaptic protein and promotes functional recovery in a rat model of traumatic brain injury. Mol Med Rep 4: 849-856, 2011.

7. Kulbatski I, Mothe AJ, Nomura $\mathrm{H}$ and Tator $\mathrm{CH}$ : Endogenous and exogenous CNS derived stem/progenitor cell approaches for neurotrauma. Curr Drug Targets 6: 111-126, 2005.

8. Galvin KA and Jones DG: Adult human neural stem cells for cell-replacement therapies in the central nervous system. Med J Aust 177: 316-318, 2002.

9. Martens DJ, Seaberg RM and Van der Kooy D: In vivo infusions of exogenous growth factors into the fourth ventricle of the adult mouse brain increase the proliferation of neural progenitors around the fourth ventricle and the central canal of the spinal cord. Eur J Neurosci 16: 1045-1057, 2002.

10. Sailor KA, Ming GL and Song H: Neurogenesis as a potential therapeutic strategy for neurodegenerative diseases. Expert Opin Biol Ther 6: 879-890, 2006.

11. Goldman SA: Directed mobilization of endogenous neural progenitor cells: the intersection of stem cell biology and gene therapy. Curr Opin Mol Ther 6: 466-472, 2004. 
12. Johansson CB, Momma S, Clarke DL, Risling M, Lendahi U and Frisen J: Identification of a neural stem cell in the adult mammalian central nervous system. Cell 96: 25-34, 1999.

13. Schwab ME: Repairing the injured spinal cord. Science 295: 1029-1031, 2002.

14. Dobkin BH and Havton LA: Basic advances and new avenues in therapy of spinal cord injury. Annu Rev Med 55: 255-282, 2004.

15. Alexanian AR, Kwob WM, Pravdic D, Maiman DJ and Fehlings MG: Survival of neurally induced mesenchymal cells may determine degree of motor recovery in injured spinal cord rats. Restor Neurol Neurosci 28: 761-767, 2010.

16. Mabie PC, Mehler MF, Marmur R, Papavasiliou A, Song Q and Kessler JA: Bone morphogenetic proteins induce astroglia differentiation of oligodendroglial-astroglial progenitor cells. J Neurosci 17: 4112-4120, 1997.

17. Mehler MF, Mabie PC, Zhu G, Gokhan S and Kessler JA: Developmental changes in progenitor cell responsiveness to bone morphogenetic proteins differentially modulate progressive CNS lineage fate. Dev Neurosci 22: 74-85, 2000.

18. Nakajima T, Ota M and Ito K: Differentiations of autonomic neurons by BMP-independent mechanisms. Cell Tissue Res 332: 25-35, 2008.

19. Blitz IL and Cho KW: Finding partners: how BMPs select their targets. Dev Dyn 238: 1321-1331, 2009.

20. Ueki T, Tanaka M, Yamashita K, et al: A novel secretory factor, Neurogenesin-1, provides neurogenic environmental cues for neural stem cells in the adult hippocampus. J Neurosci 23 11732-11740, 2003.

21. Sakuta H, Suzuki R, Takahashi H, et al: Ventroptin: a BMP-4 antagonist expressed in a double-gradient pattern in the retina. Science 293: 111-115, 2001.

22. Yin ZS, Zhang H, Wang W, Hua XY, Hu Y, Zhang SQ and Li GW: Wnt-3a protein promote neuronal differentiation of neural stem cells derived from adult mouse spinal cord. Neurol Res 29: 847-854, 2007.

23. Xiao Q, Du Y, Wu W and Yip HK: Bone morphogenetic proteins mediate cellular response and, together with Noggin, regulate astrocyte differentiation after spinal cord injury. Exp Neurol 221: $353-366,2010$
24. Nakashima K, Takizawa T, Ochiai W, et al: BMP2-mediated alteration in the developmental pathway of fetal mouse brain cells from neurogenesis to astrocytogenesis. Proc Natl Acad Sci USA 98: 5868-5873, 2001

25. Yanagisawa M, Takizawa T, Ochiai W, Uemura A, Nakashima K and Taga T: Fate alternation of neuroepithelial cells from neurogenesis to astrocytogenesis by bone morphogenetic proteins. Neurosci Res 41: 391-396, 2001.

26. Mehler MF, Mabie PC, Zhang D and Kessler JA: Bone morphogenetic proteins in the nervous system. Trends Neurosci 20: 309-317, 1997.

27. Li W, Cogswell CA and LoTurco JJ: Neuronal differentiation of precursors in the neocortical ventricular zone is triggered by BMP. J Neurosci 18: 8853-8862, 1998.

28. Mabie PC, Mehler MF and Kessle JA: Multiple roles of bone morphogenetic protein signaling in the regulation of cortical cell number and phenotype. J Neurosci 19: 7077-7088, 1999.

29. Lim DA, Tramontin AD, Trevejo JM, Herrera DG, Garcia-Verdugo JM and Alvarez-Buylla A: Noggin antagonizes BMP signaling to create a niche for adult neurogenesis. Neuron 28: 713-726, 2000.

30. Shakèd M, Weissmüller K, Svoboda H, Hortschansky P, Nishino N, Wölfl S and Tucker KL: Histone deacetylases control neurogenesis in embryonic brain inhibition of BMP2/4 signaling. PLoS One 3: e2668, 2008.

31. Weible MW II and Chan-Ling T: Phenotypic characterization of neural stem cells from human fetal spinal cord: synergistic effect of LIF and BMP4 to generate astrocytes. Glia 55: 1156-1168, 2007.

32. Sasai Y: Regulation of neural determination by evolutionarily conserved signals: anti-BMP factors and what next? Curr Opin Neurobiol 11: 22-26, 2001.

33. Fu SL, Ma ZW, Yin L, Iannotti C, Lu PH and Xu XM: Region-specific growth properties and trophic requirements of brain- and spinal cord-derived rat embryonic neural precursor cells. Neuroscience 135: 851-862, 2005. 